\title{
THE IMPACT OF THE CURING AND POST-STRETCHING PROCESS ON THE STRESSES DISTRIBUTION AROUND AN OPEN HOLE IN GLARE LAMINATE
}

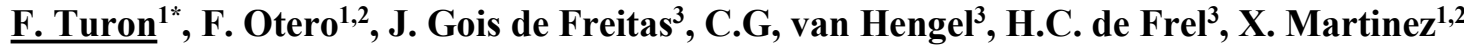 \\ ${ }^{1}$ International Center for Numerical Methods in Engineering (CIMNE) \\ Technical University of Catalonia (UPC) \\ Gran Capitn s/n, 08034 Barcelona, Spain \\ ${ }^{2}$ Departamento de Ciencia e Ingeniera Nutica, FNB \\ Technical University of Catalonia (UPC) \\ Pla de Palau 18, 08003 Barcelona, Spain \\ ${ }^{3}$ GKN Fokker Aerostructures \\ Industrieweg 4, 3351 LB Papendrecht, The Netherlands \\ *fturon@cimne.upc.edu
}

Glass Laminate Aluminum Reinforced Epoxy (GLARE) is a laminate within the Fibre Metal Laminates (FML) family which consists in a combination of very thin aluminium sheets and composite glass-fibre/epoxy-resin layers. This FML is widely used in the aeronautical sector because, compared to aluminium material, it provides a better structural strength and fatigue resistance, along with a better impact and corrosion resistance.

However, one drawback of GLARE are the residual stresses obtained during its manufacturing. The high temperatures applied to the material during the resin curing process, required also to bond the composite and the metal, contract the layers within the laminate. The temperature at which this occurs is known as the stress-free temperature [1]. This bonding, together with the subsequent cooling of the laminate, results in residual stresses caused by the different thermal coefficient of each material. To minimize these stresses, the material is subjected to an after curing post-stretching process. The purpose of the post-stretching is to modify the stress balance within the GLARE layers in order to improve the laminate mechanical properties. This is achieved thanks to the plastic behaviour of the aluminium. Generally, at the end of the curing, aluminium layers remain under tensile stress but if a permanent deformation is applied, this tensile load can be turned into a compression. As a consequence, the mechanical behaviour of GLARE material is improved, when subjected to cyclic loads, improving its fatigue performance. Finally, and quite often, GLARE panels are machined, which adds new residual stresses to the material.

This work presents the numerical analysis of the manufacturing process of a GLARE panel, and its further structural modification by machining with an open hole. The analysis made obtains the material performances by means of Serial Parallel mixing theory (SP RoM) [2]. The behaviour of the unidirectional Glass Fibre Reinforced Polymer (GFRP) is defined using a damage model for the epoxy and an elastic model for the fibre. Aluminium is simulated with a plasticity model. With the proposed approach, the simulation is capable of obtaining the stress state of GLARE components in the different phases of the manufacturing process: after curing, post-stretching and machining a hole in it. The analysis made will also apply a final stretch to the specimen in order to evaluate the performance of the material once it is in service. Results obtained are compared with experimental data from [1], showing good agreement. This simulation capability will allow improving the whole manufacturing process, in order to minimize the residual stresses in the material and its final mechanical performance. 


\section{References}

[1] E. Kappela, R. Prussaka, J. Wiedemann (2019) On a simultaneous use of fiber-Bragggratings and strain-gages to determine the stress-free temperature Tsf during GLARE manufacturing. Composite Structures, 227, 111279.

[2] F. Rastellini, S. Oller, O. Salomon, E. Onate (2008) Composite materials non-linear modelling for long fibre-reinforced laminates continuum basis, computational aspect and validations. Computers and Structures. 86, 879-896.
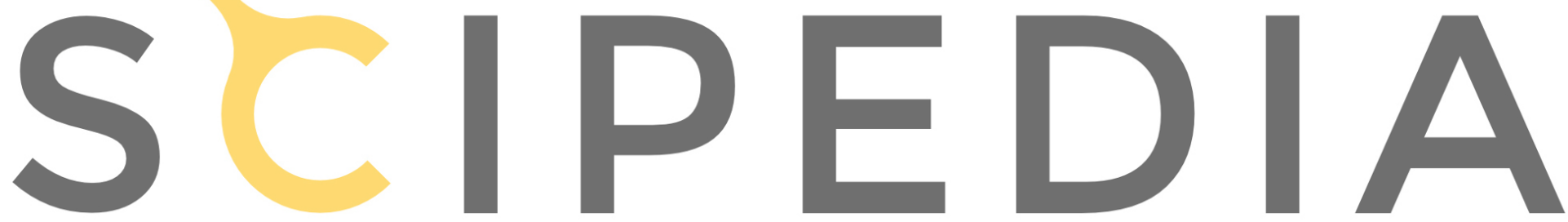